\title{
Love at the time of the Covid-19 pandemic: preliminary results of an online survey conducted during the quarantine in Italy
}

\author{
Andrea Cocci $\mathbb{D}^{1}$ - Daniel Giunti ${ }^{2}$ - Camilla Tonioni ${ }^{2} \cdot$ Giovanni Cacciamani $^{3} \cdot$ Riccardo Tellini $^{1} \cdot$ Gaia Polloni $^{4}$. \\ Gianmartin Cito $\mathbb{D}^{1} \cdot$ Fabrizio Presicce $e^{5}$ Marina Di Mauro $\mathbb{D}^{6} \cdot$ Andrea Minervini $^{1} \cdot$ Sebastiano Cimino ${ }^{6}$. \\ Giorgio Ivan Russo $\mathbb{1}^{6}$
}

Received: 26 April 2020 / Revised: 4 May 2020 / Accepted: 5 May 2020 / Published online: 14 May 2020

(c) The Author(s), under exclusive licence to Springer Nature Limited 2020

\section{To the Editor:}

The ongoing pandemic of the Coronavirus infection (COVID-19) and its restrictive measures may lead to widespread fear, anxiety and panic, which can escalate into further negative psychological reactions, including adjustment disorder, depression and changes in sexual behaviour [1]. Clinical depression is often associated with a reduction in sexual interest and sexual response, an association that may be more marked in women than in men [2].

In this report, we present the preliminary results of an online survey conducted during the quarantine in Italy. We aimed to assess the impact of the quarantine due to COVID-19 on psychological (in particular anxiety and depression symptoms) and sexual well-being. The sample was drawn through posts on social networks (Instagram and Facebook ${ }^{\circ}$ ) and the survey was administered online through Google Forms. Participants completed the survey with questions about their sexual habits they had before and during the quarantine (Table 1). The Beck Depression

Supplementary information The online version of this article (https:// doi.org/10.1038/s41443-020-0305-x) contains supplementary material, which is available to authorized users.

Giorgio Ivan Russo

giorgioivan1987@gmail.com

Department of Urology, University of Florence, Florence, Italy

2 Centro Integrato di Sessuologia Il Ponte, Florence, Italy

3 USC Institute of Urology and Catherine and Joseph Aresty Department of Urology, University of Southern California, Los Angeles, CA, USA

4 Private Psychologist, Sapienza University, Rome, Italy

5 Department of Urology, Sapienza University, Rome, Italy

6 Urology Section, Department of Surgery, University of Catania, Catania, Italy
Inventory (BDI-II) [3] in its Italian validated version [4] and the Beck Anxiety Inventory (BAI) [5] in the Italian validated version [4] were used. Moreover, sexual satisfaction was evaluated by asking the question "Overall, how satisfied are you with your sex life during quarantine on a scale ranging from 1 (not at all satisfied) to 5 (extremely satisfied)?" and people were asked "Have you been quarantined as positive for COVID-19?". The survey also investigated items about autoerotism during and before quarantine. Supplementary Table 1 shows the questions of the survey.

A total of 1515 respondents were involved in the study. None of the participants were quarantined as positive for COVID-19. Median age was 21.0 (interquartile range [IQR]: 19.0-25.0), median BDI score was 10.0 (IQR: 5.0-16.0) and median BAI score was 11.0 (IQR: 6.0-20.0). Table 1 shows sexual activity domains before and during the quarantine. Moreover, 602 (39.74\%) respondents answered that quarantine increased autoerotism more than before. Although only $7.46 \%$ of the participants reported "no satisfaction" before the quarantine, 53.53\% complained about that during the quarantine $(p<0.01)$. We found that women had greater depression (BDI-male: 8.0 [IQR 4.0-13.0]; BDI-female: 11.0 [IQR 6.0-17.0]; $p<0.01$ ) and anxiety respect to male (BAI-male: 7.0 [IQR 3.0-14.0]; BAI-female 13.0 [IQR $7.0-23.0] ; p<0.01)$. At the multivariate logistic regression analysis, we found that age (odds ratio [OR]: 0.96; $p<0.01)$ and BDI (OR: $1.07 ; p<0.01)$ in male and age (OR: $0.96 ; p<0.01$ ), BDI (OR: $1.03 ; p<0.01$ ) and "knowing people positive at COVID" (OR: $0.78 ; 0.05$ ) in women were significant predictors of sexual dissatisfaction.

We interestingly found that more than $40 \%$ of the respondents reported an increased sexual desire during the quarantine, compared to baseline. However, the reported enhanced sex arousal did not translate into a higher 
Table 1 Sexual activity domains before and during quarantine.

$p$ value

Sexual intercourse during quarantine, $n(\%)$

$\begin{array}{lrl}\text { Yes } & 239(15.78) & P<0.01^{\mathrm{a}} \\ \text { No } & 1276(84.22)\end{array}$

Did you have intercourse before quarantine? $n(\%)$

Yes

No

$1231(81.25)$

Number of intercourses, $n(\%)$

0

$1276(84.22)$

1 per week

157 (10.36)

2 per week

$50(3.30)$

$>2$ per week

$32(2.11)$

Sexual desire during quarantine, $n(\%)$

No sexual desire

$241(15.91)$

Less than before

Equal to before

594 (39.21)

More than before

616 (40.66)

Do you practice autoerotism? $n(\%)$

Yes

1195 (78.88)

No

$320(21.12)$

How did autoerotism change during quarantine? $n(\%)$

No autoerotism

$320(21.12)$

Less than before

$147(9.70)$

Equal

446 (29.44)

More than before

$602(39.74)$

Do you see porn movies? $n(\%)$

Yes

$$
P<0.01^{\mathrm{a}}
$$

$921(60.79)$

No

Did you see porn movies before quarantine? $N(\%)$

Yes

986 (65.08)

529 (34.92)

Sexual satisfaction during quarantine, $n(\%)$

No satisfaction

$811(53.53)$

Mild satisfied

369 (24.36)

Equally satisfied

171 (11.29)

Moderate satisfied

107 (7.06)

57 (3.76)

Extremely satisfied

$113(7.46)$

149 (9.83)

277 (18.28)

530 (34.98)

446 (29.44)

Extremely satisfied

${ }^{\mathrm{a} C h i}$ square test

frequency of sexual intercourse ( $18.75 \%$ and $15.78 \%$ before and during quarantine, respectively). Moreover, sexual satisfaction significantly decreased during the quarantine, with more than half of the respondents describing a complete absence of sexual satisfaction, compared with only $7.46 \%$ of people before the lockdown. These results can be partly explained by a self-reported increase of autoerotism in $40 \%$ of the participants and a high prevalence of pornography use between the responders; in fact, it is widely accepted that pornography use is associated with decreased sexual satisfaction [6].

The limitations of this online survey include the sampling process, the lack of validated questionnaires about sexual activity and the recall bias for questions assessing sexual function before the quarantine. However, this survey is one of the few reports evaluating the impact of quarantine on sexuality of males and females. We found that lower age and higher depression score were significant predictors of sexual dissatisfaction in both genders. Interestingly, we found that women who knew patients positive for COVID-19 were at a reduced risk of sexual satisfaction, may be due to the mitigation of stress secondary to a better knowledge of the disease itself. Further analyses and longer follow-up after the quarantine are required in order to confirm these preliminary results and to investigate different changes in sexual health.

\section{Compliance with ethical standards}

Conflict of interest The authors declare that they have no conflict of interest.

Publisher's note Springer Nature remains neutral with regard to jurisdictional claims in published maps and institutional affiliations.

\section{References}

1. Cocci A, Presicce F, Russo GI, Cacciamani G, Cimino S, Minervini A. How sexual medicine is facing the outbreak of COVID-19: experience of Italian urological community and future perspectives. Int J Impot Res. 2020. https://doi.org/10.1038/ s41443-020-0270-4.

2. Angst J. Sexual problems in healthy and depressed persons. Int Clin Psychopharmacol. 1998;13:S1-4.

3. Beck AT, Steer RA, Ball R, Ranieri WF. Comparison of Beck depression inventories-IA and-II in psychiatric outpatients. J Pers Assess. 1996;67:588-97.

4. Sica C, Ghisi M. The Italian versions of the Beck Anxiety Inventory and the Beck Depression Inventory-II: psychometric properties and discriminant power. In: Lange MA, editor. Leadingedge psychological tests and testing research. Hauppauge, New York: NOVA Science; 2007. p. 27-50.

5. Beck AT, Epstein N, Brown G, Steer RA. An inventory for measuring clinical anxiety: psychometric properties. J Consult Clin Psychol. 1988;56:893-7.

6. Dwulit AD, Rzymski P. The potential associations of pornography use with sexual dysfunctions: an integrative literature review of observational studies. J Clin Med. 2019;8. https://doi.org/10.3390/ jem8070914. 